\title{
The K. Thomas - R. Kilmann \\ questionnaire: the vulnerabilities \\ of the diagnostic tool
}

\section{A. S. Melnichuk ${ }^{1}$}

${ }^{1}$ RANEPA, 33 Zhelyabova str., Tver, 170100, Russian Federation

DOI: 10.18255/1996-5648-2021-4-630-637

Research article

Full text in Russian

The results of the analysis of the text of the $\mathrm{K}$. Thomas $-\mathrm{R}$. Kilmann questionnaire are presented. It is shown that the formulation of many items is not specific for their unambiguous assignment to the prescribed scales. In some formulations, the context of the mode of behavior that is proposed to be chosen is not clearly defined. In many of the pairs of statements, the options are difficult to distinguish from each other or are not strictly alternative. The directions of work for overcoming the identified vulnerabilities of the questionnaire are proposed.

Keywords: K. Thomas - R. Kilmann Questionnaire; style of conflict behavior; psychometric characteristics

\section{INFORMATION ABOUT AUTHORS}

Melnichuk, Andrey S.. E-mail: melnichook@rambler.ru

Cand. Sc (Psychology), AssociateProfessor 


\title{
Опросник К. Томаса - Р. Килманна: уязвимости диагностического инструмента
}

\author{
А. С. Мельничук ${ }^{1}$
}

${ }^{1}$ Российская академия народного хозяйства и государственной службы при Президенте РФ, ул. Желябова, 33, Тверь, 170100, Российская Федерация

DOI: 10.18255/1996-5648-2021-4-630-637

УдК 159.9.072.432

Научная статья

Представлены результаты анализа текста опросника К. Томаса Р. Килманна. Показано, что фрормулировки многих пунктов не специфичны для их однозначного отнесения к предписанным шкалам. В некоторых фрормулировках четко не определен контекст способа поведения, который предлагается выбрать. Во многих парах высказываний варианты трудно отличимы друг от друга или не являются строго альтернативными. Предложены направления работы по преодолению выявленных уязвимостей опросника.

Ключевые слова: опросник К. Томаса - Р. Килманна; стиль; поведение в конфликте; психометрические характеристики

\section{ИНФОРМАЦИЯ ОБ АВТОРАХ}

Мельничук, Андрей Степанович $\mid$ E-mail: melnichook@rambler.ru

Кандидат психологических наук, доцент

\section{Постановка проблемы}

Социальное взаимодействие невозможно без столкновения мнений, интересов или намерений людей. Это определяет большую научную и практическую значимость изучения конфликтного поведения и его стилевых характеристик. Среди инструментов, предназначенных для оценки данного явления, выделяется опросник, разработанный У. Томасом и Р. Килманном в начале $1970-x$ гг. [1] и до сих пор остающийся одним из наиболее используемых [например, 2-3].

За рубежом достаточно широко применяются и иные диагностические средства, например опросник М. А. Рахима [4]. В этом контексте тест Томаса ${ }^{1}$ занимает в нашей стране особое место, являясь, по существу, единственной методикой для диагностики стиля поведения в конфликте (в данном

${ }^{1}$ Несмотря на наличие у методики двух авторов, в отечественных публикациях она, как правило, обозначается как «тест / методика Томаса». Исходя из этой традиции, а также для краткости мы в данной статье также будем использовать такое наименование.

() Мельничук А. С., 2021

Статья открытого доступа под лицензией CC BY (https://creativecommons.org/licenses/by/4.0/) 
Мельничук А. С.

случае мы не рассматриваем оригинальные инструменты сходного назначения, разрабатываемые авторами для своих собственных исследований и гораздо менее известные, например методику «Конфликтные ситуации» [5]).

Следует констатировать парадоксальную ситуацию. С одной стороны, методика Томаса в течение нескольких десятилетий интенсивно используется в отечественной науке и практике. Для большинства исследователей она имеет статус «классического» инструмента, в отношении которого вопрос о его качестве не возникает "по определению». С другой стороны, как справедливо было отмечено С. В. Кардашиной и Н. В. Шаньгиной, отсутствуют какие-либо данные о психометрических характеристиках теста Томаса, как и инорормация о его российской адаптации (причем приводимые в публикациях переводы пунктов имеют отличия от англоязычного текста) [6].

Статья С. В. Кардашиной и Н. В. Шаньгиной - единственная отечественная публикация, в которой рассмотрены психометрические показатели теста. В ней приведены полученные У. Томасом и Р. Килманном данные о её валидности, надежности, влиянии социальной желательности. Весьма ценным стал анализ согласованности шкал русскоязычного варианта теста, осуществленный на основе опроса объемной и гетерогенной выборки. По итогам исследования был сделан вывод о достаточно высокой внутренней валидности методики, а также предложены уточненные фрормулировки перевода некоторых пунктов (более корректно отражающие смысл исходного текста) и тестовые нормы.

Не менее значимым представляется рассмотрение пунктов теста Томаса с точки зрения их содержания. Представленная в статье С. В. Кардашиной и Н. В. Шаньгиной группировка утверждений опросника по шкалам позволила заметить ряд проблемных мест, снижающих валидность методики. Их наличие было подтверждено в ходе проведенного нами специального рассмотрения текста опросника Томаса.

\section{Анализ результатов исследования}

Наиболее корректными являются пункты шкалы «Компромисс», адекватно отражающие сущность данного стиля. В рамках шкалы «Соперничество» проблемными представляются пункты 14Б и 25А («Я пытаюсь показать другому логику и преимущества моих взглядов»), так как попытка изложить свою точки зрения не специфрчна для соперничества, а присуща любому диалогу (в том числе при компромиссе и сотрудничестве).

Уязвимости присутствуют и в рамках шкалы «Сотрудничество». Во-первых, это пункты 8Б, 11А и 19А («Я первым делом стараюсь ясно определить то, в чем состоят все затронутые интересы и вопросы»). Четкое выявление поля конфликта не имеет отношения исключительно к сотрудничеству, так как понимание «вокруг чего начался спор» - важный момент 
любого поведения в контексте конфликта. Это потенциально может приводить к трудностям респондентов при необходимости делать выбор между пунктами 8Б и 8А, отражающими стиль соперничества.

Не специфричен для сотрудничества пункт 21Б («Я всегда склоняюсь к прямому обсуждению проблемы»), описывающий достаточно общий подход к коммуникации в ситуации разногласий. Во-вторых, не вполне корректны пункты 23А и 26Б, отражающие озабоченность «тем, чтобы удовлетворить желания каждого из нас», так как нет указания на то, насколько полно предполагается это сделать. Поэтому они могут описывать также и стиль компромисса. Для характеристики сотрудничества более уместен перевод С. В. Кардашиной и Н. В. Шаньгиной, в котором говорится об удовлетворении именно «всех наших пожеланий». Аналогичная ситуация имеет место и с пунктами, которые по своей формулировке могут характеризовать стили как сотрудничества, так и компромисса, - 2Б («Я пытаюсь уладить дело с учетом интересов другого и моих собственных») и 30Б («Я всегда занимаю такую позицию в спорном вопросе, чтобы мы совместно с другим заинтересованным человеком могли добиться успеха»).

Пункты 5А и 28Б могут вызвать у респондентов трудности при ответе в силу размытости контекста указанного в них поведения - стремления «искать поддержку у другого». Во-первых, не ясно, какая именно поддержка имеется в виду - интеллектуальная, эмоциональная и т. д. Во-вторых, термин «другой» может пониматься как собственно оппонент, член своей «группы поддержки» или как «третья сторона». Наконец, не ясно отнесение к стилю сотрудничества пункта $20 \mathrm{~A} \mathrm{(«Я} \mathrm{пытаюсь} \mathrm{немедленно} \mathrm{преодолеть}$ наши разногласия»), так как не указано, как именно будет решаться спорная ситуация.

В шкале «Избегание» присутствуют пункты 5Б, 15Б и 17Б, характеризующие стремление сделать все возможное, чтобы «избежать [бесполезной] напряженности». Мы полагаем, что следует разделять ориентацию на недопущение эскалации противоборства и собственно предпочтение стиля избегания (как склонность ухода от противоборства в принципе). Указанные пункты методики связаны не только со стилем избегания, они могут характеризовать стили сотрудничества, компромисса и даже уступки.

Пункт 6А («Я пытаюсь избежать возникновения неприятностей для себя») также не специфичен для характеристики избегания, так как реализовать такую интенцию можно с помощью иных стратегий, например приспособления. Кроме того, термин «неприятности» весьма широк. Это потенциально дает возможность говорить об их предотвращении даже с помощью соперничества, если неприятности будут связаны именно с проигрышем в споре. В свете этого обратим внимание, что в вопросе № 6 респондент как раз и должен сделать выбор между фрормулировкой 6 А и 6Б, характеризующей именно соперничество («Я стараюсь добиться своего»), но такой выбор отнюдь не является строго альтернативным. 
Смысловая размытость присуща и пунктам 1А и 23Б («Иногда я предоставляю возможность другим взять на себя ответственность за решение спорного вопроса»), так как не ясно, что следует понимать под ответственностью и почему такое поведение ассоциируется именно с избеганием (ведь человек, по сути, уже вовлечен в конфликт).

Определенные вопросы вызывают пункты 12A и 27A («Зачастую я избегаю занимать позицию, которая может вызвать споры»). Такая формулировка, несомненно, важна для диагностики уровня общей конфликтности личности. Но, если рассматривать тест Томаса как инструмент, оценивающий предпочтения в уже возникшем противостоянии, она не имеет смысл (противоречие позиций уже имеет место, но человек не стремится в него вовлекаться). Для характеристики стиля избегания более уместной представляется высказывание вида «при возникновении спорных вопросов я предпочитаю уйти от их обсуждения и возможного столкновения».

Неоднозначны и пункты 9А и 29A («Думаю, что не всегда стоит волноваться из-за каких-то возникающих разногласий»). Представляется, что указанное высказывание характеризует не собственно привычное поведение в ситуации конфликта, а эмоциональные «измерения» такого поведения (которое само по себе может быть различным). Спокойное отношение к разногласиям не только снижает вероятность втягивания в противоборство (и в этом плане связано со стилем избегания), но и выступает условием продуктивной реализации стратегий компромисса или сотрудничества.

Ряд проблемных мест присутствует и в шкале «Приспособление». Так, три её пункта (3Б, 11Б и 15А) отражают стремление «успокоить другого и главным образом сохранить наши отношения». Ориентация на сохранение отношений - существенная черта «уступающего» стиля. Однако в указанной фрормулировке явно не сказано, что ради этого человек готов жертвовать своими интересами. Кроме того, стремление снизить накал эмоций вполне сочетается с ориентацией на поиск компромисса или сотрудничество. По сути, в указанной формулировке соединены два достаточно разных аспекта поведения - характер ориентации на свои / чужие интересы и предпочитаемая степень эмоциональности диалога. Недостаточная специфичность характерна и для ряда других высказываний рассматриваемой шкалы. Так, стремление «не задеть чувств другого» / «не обижать партнеров» (пункты 16A и 30A) не столько характеризует склонность к уступкам, сколько выступает фрактором создания атмосферы, благоприятной для достижений компромисса или налаживания сотрудничества.

То же самое относится и к таким пунктам, как 21А и 25Б («Ведя переговоры, я стараюсь быть внимательным к желаниям другого). Такая интенция может не только сопровождать предпочтение стиля приспособления, но и стать значимым элементом ориентации на сотрудничество и компромисс (предполагающие учет интересов оппонента). Для более корректного отнесения обсуждаемых пунктов к шкале «Приспособление» было бы умест- 
нее сорормулировать их следующим образом: «Ведя переговоры, я в большей мере стараюсь учесть интересы партнера, чем свои собственные».

Отраженная в пункте 1Б склонность в первую очередь «обращать внимание на то, с чем мы оба согласны» имеет сомнительную связь именно со стилем приспособления (как ориентации на интересы другого). Одновременно указанная фрормулировка хорошо характеризует стремление к созданию психологической общности сторон, крайне важной в контексте ориентации на сотрудничество и компромисс.

Помимо сказанного выше, следует учитывать, что методика Томаса предполагает в каждом вопросе выбор одного из двух высказываний. Условием корректности такого выбора являются отличимость вариантов друг от друга (не допускающая смыслового смешения) и их явная альтернативность. В ряде мест методики эти правила не соблюдаются в полной мере.

Например, в пункте № 2 респонденту предлагается сделать выбор между высказываниями: «Я стараюсь найти компромиссное решение» (шкала компромисса) и «Я пытаюсь уладить дело с учетом интересов другого и моих собственных» (шкала сотрудничества). Можно увидеть, что эти формулировки имеют очень близкий смысл. То же самое следует отнести и к другой паре высказываний, репрезентирующих эти же шкалы - $26 \mathrm{~A}$ («Я предлагаю среднюю позицию») и 26Б («Я почти всегда озабочен тем, чтобы удовлетворить желания каждого из нас»), поскольку степень удовлетворения желаний явно не указана и может совпадать с 50/50.

В текущих фрормулировках также весьма близки высказывания, призванные отразить стиль приспособления (18А - «Если это сделает другого счастливым, я дам ему возможность настоять на своем») и компромисса (18Б - «Я даю возможность другому в чем-то остаться при своем мнении, если он также идет мне навстречу»). Сходство обусловлено тем, что компромисс по своей сути предполагает некоторые уступки, а в пункте 18Б нет четкого указания на пределы «настаивания на своем»

Некоторые сопоставляемые пары высказываний оказываются взаимно предполагающими или взаимно дополняющими друг друга. Так, в пункте № 21 отнесенное к шкале приспособления высказывание А («Ведя переговоры, я стараюсь быть внимательным к желаниям другого») отнюдь не исключает высказывания Б, принадлежащего к шкале сотрудничества («Я всегда склоняюсь к прямому обсуждению проблемы»).

Не является строго альтернативным также выбор между пунктами шкалы сотрудничества (11А - «Первым делом я стараюсь ясно определить то, в чем состоят все затронутые интересы и вопросы») и приспособления (11Б - «Я стараюсь успокоить другого и главным образом сохранить наши отношения»). Для проходящего диагностику человека вполне может быть характерна одновременная ориентация как на прояснение интересов и позиций сторон, так и на психологическую поддержку партнера в целях пролонгирования взаимодействия. 
Мельничук А. С.

Взаимно дополняющими оказываются и другие пункты, принадлежащие к этой же паре шкал, - соответственно 30Б («Я всегда занимаю такую позицию в спорном вопросе, чтобы мы совместно с другим заинтересованным человеком могли добиться успеха») и $30 \mathrm{~A}$ («Я стараюсь не задеть чувств другого»). Мы исходим из того, что уважение к переживаниям оппонента - это одна из предпосылок совместного решения проблемы.

В пункте № 15 «сталкиваются» высказывания «Я стараюсь успокоить другого и главным образом сохранить наши отношения» (шкала приспособления) и «Я стараюсь сделать все необходимое, чтобы избежать напряженности» (шкала избегания). Но предотвращение роста напряженности в отношениях неразрывно связано с действиями по снижению уровня напряженности у партнера.

Как это ни парадоксально, но возможны сложности даже при выборе между индикаторами стиля сотрудничества (14A - «Я сообщаю другому свою точку зрения и спрашиваю о его взглядах») и соперничества (14Б - «Я пытаюсь показать другому логику и преимущества моих взглядов»). Трудность здесь обусловлена тем, что присутствующее в первом высказывании изложение своей позиции вполне может сочетаться с аргументацией её справедливости.

\section{Выводы}

Обобщая все сказанное, следует отметить противоречивую ситуацию с методикой Томаса. С одной стороны, она имеет несомненные достоинства, связанные с ясностью, лежащей в её основе концепции, небольшим объемом, простотой заполнения, обработки ответов и интерпретации результатов. С другой стороны, можно говорить о наличии в тексте методики достаточно существенных «уязвимостей», способных существенно влиять на валидность получаемых данных.

Во-первых, в ряде формулировок недостаточно четко указан контекст предъявляемых ситуаций. Помимо сложности для понимания, это может вести к несовпадающей трактовке пунктов различными респондентами (и, соответственно, к некорректности обобщения получаемых результатов).

Во-вторых, к числу уязвимостей следует отнести присутствующую в ряде вопросов недостаточную альтернативность высказываний и их смысловое пересечение, способные повлиять на результаты выбора ответов.

В-третьих, это недостаточно четкое соответствие входящих в шкалы пунктов смыслу оцениваемых этими шкалами стилей поведения в конфрликте. Смешение пунктов (которые теоретически должны были относиться к разным шкалам) может быть связано с наличием способов реагирования на конфрликты, которые не были предусмотрены заложенной в методику моделью. Такие способы по отношению к шкалам методики являются «дополняющими», т. е. могут применяться для описания одновременно нескольких стилей поведения. 
Можно выделить несколько направлений работы по преодолению указанных уязвимостей методики Томаса.

Первое направление ориентировано на внесение изменений при сохранении принципов построения и объема исходной методики. Коррективы могут включать переформулировку проблемных пунктов с целью большего соответствия сущности шкал, а также создание более удачных пар высказываний (в том числе путем перестановки прежних или переформулированных пунктов). Например, относящийся к шкале соперничества пункт «Я пытаюсь продемонстрировать партнеру логику и выгоды моей позиции» было бы точнее сформулировать как «Я пытаюсь продемонстрировать партнеру логику и выгоды толъко / именно моей позиции».

Второе направление предполагает внесение более существенных изменений (в силу чего корректнее вести речь уже о разработке нового инструмента, функционально и идеологически сходного с методикой Томаса). Такая работа может, например, включать исключение особенно проблемных пар пунктов с общим сокращением объема методики (это представляется возможным в силу того, что каждый из стилей сочетается с другими по три раза).

Третье направление предполагает максимальный отход от существующего построения методики Томаса и может заключаться в использовании иных ипсативных приемов при сохранении существующей типологии стилей или же при включении в неё дополнительных стилевых параметров.

\section{Ссылки}

1. Kilmann R. H., Thomas K. W. Developing a Forced-Choice Measure of ConflictHandling Behaviour: The «MODE» Instrument // Educational and Psychological Measurement. 1977. Vol. 37, №. 2. P. 309-325.

2. Altmäe S., Türk K., Toomet O.-S. Thomas-Kilmann's Conflict Management Modes and their relationship to Fiedler's Leadership Styles (basing on Estonian organizations) // Baltic Journal of Management. 2013. Vol. 8, № 1. P. 45-65.

3. Gupta S., Bhardwaj S. A study on the relationships between MBTI psychological types and MODE conflict styles // Asian Journal Of Management Research. 2014. Vol. 5, № 1. P. 31-43.

4. Rahim M. A Managing conflict in organizations. Westport. Quorum Books, 2001. $309 \mathrm{p}$.

5. Синельникова Е. С. Эмоциональный интеллект как фрактор взаимодействия в конфликте: кросс-культурный аспект: дис. ... канд. психол. наук. СПб., 2015. 195 с.

6. Кардашина С. В., Шаньгина Н. В. Психометрические характеристики русскоязычной версии Опросника К. Томаса - P. Килманна («Thomas - Kilmann Conflict Mode Instrument - TKI-R») // Педагогическое образование в России. 2016. № 11. C. 216-228. 